\title{
Effects of pre-analytical storage time, temperature, and freeze-thaw times on coagulation factors activities in citrate-anticoagulated plasma
}

\author{
Ying Zhao ${ }^{1}$, Guofang Feng ${ }^{2}$, Limin Feng ${ }^{1}$ \\ ${ }^{1}$ Department of Clinical Laboratory, The First Affiliated Hospital, College of Medicine, Zhejiang University, Hangzhou 310003, China; ${ }^{2}$ Women's \\ Hospital, School of Medicine, Zhejiang University, Hangzhou 310006, China \\ Contributions: (I) Conception and design: L Feng, Y Zhao; (II) Administrative support: L Feng; (III) Provision of study materials or patients: All \\ authors; (IV) Collection and assembly of data: All authors; (V) Data analysis and interpretation: L Feng, Y Zhao; (VI) Manuscript writing: All \\ authors; (VII) Final approval of manuscript: All authors. \\ Correspondence to: Limin Feng. Department of Clinical Laboratory, The First Affiliated Hospital, College of Medicine, Zhejiang University, Qingchun \\ Road No. 79, Hangzhou 310003, China. Email: FLM@zju.edu.cn.
}

Background: Coagulation factor assays are very important for diagnosing, treating, and monitoring
inherited and acquired factor deficiencies. Appropriate pre-analytical storage conditions of citrate-
anticoagulated plasma are essential for detection of coagulation factor activity. We aimed to investigate the
effects of storage temperature and time on coagulation factor (F) II, FV, FVII, FX, FXI, and FXII activity up
to $24 \mathrm{~h}$ and the effects of freeze-thaw times at $-80^{\circ} \mathrm{C}$ on factor activity.
Methods: Twenty-two blood samples were analyzed after storage for 0 (baseline), $2,4,6,8,12$, and 24 h at
25 and $4{ }^{\circ} \mathrm{C}$. Mean percent changes, numbers of samples with $>10 \%$ changes, percent change trend plots, and
difference plots were evaluated to determine clinically relevant differences.
Results: The acceptable storage times for FII coagulation activity (FII:C), FV:C, FVII:C, FX:C, FXI:C,
and FXII:C were $24,8,8,24,12$, and $12 \mathrm{~h}$ at $4{ }^{\circ} \mathrm{C}$ and $24,4,8,8,12$, and $12 \mathrm{~h}$ at $25{ }^{\circ} \mathrm{C}$, respectively. The
acceptable freeze-thaw times for FII:C, FV:C, FVII:C, FX:C, FXI:C, and FXII:C were $2,2,3,3,2$, and 1 ,
respectively.

Conclusions: When factor activity cannot be determined within these acceptable timeframes, we recommend that plasma samples should be frozen and thawed at appropriate times for analysis.

Keywords: Coagulation factor; mean percent changes; storage temperature; freeze-thaw study; acceptable timeframes

Submitted May 20, 2018. Accepted for publication Nov 05, 2018.

doi: 10.21037/atm.2018.11.24

View this article at: http://dx.doi.org/10.21037/atm.2018.11.24

\section{Introduction}

Coagulation factor activity assays are very important for diagnosing inherited and acquired factor deficiencies (1-3). Inherited factor deficiencies affect the intrinsic and extrinsic blood coagulation pathways and may lead to lifelong bleeding disorders (1-3). The severity of these disorders tends to be proportional to the degree of factor deficiency. Inherited factor (F) II, FV, FVII, FX, FXI, and FXII deficiencies are rare autosomal recessive coagulation disorders with asymptomatic, moderate, or severe bleeding tendencies (4-11). The liver is the primary production site for most coagulation factors, and thus acquired factor deficiencies are closely related to the liver $(12,13)$. Diagnosis of single factor and/or combined clotting factor defects can be difficult using routine coagulation tests, and only specific factor assays allow the correct diagnosis to be reached. Meanwhile, factor assays are essential for the treatment and monitoring of bleeding disorders and thrombosis $(13,14)$. 
With the development of a hierarchical medical system in China, some patients requiring treatment for bleeding disorders or hepatic diseases consult doctors in small hospitals, community hospitals, and clinics, or family doctors $(15,16)$. As coagulation factor assays are not carried out in many hospitals in China, the samples collected from these patients are sent to independent clinical laboratories or certain comprehensive hospitals for analysis (17). The delivery time of such samples often exceeds $4 \mathrm{~h}$. The Clinical and Laboratory Standards Institute H21-A5 guideline does not clearly specify appropriate storage times and temperatures for coagulation factor assays, unlike the case for other assays in the guideline, such as the recommendation that samples for other assays should be centrifuged and tested within $4 \mathrm{~h}$ at room temperature after collection (18). Furthermore, the guideline recommends that if such samples cannot be analyzed within $4 \mathrm{~h}$, the plasma should be removed from the cellular component, and if the samples cannot be analyzed within $24 \mathrm{~h}$, the plasma should be removed without disturbing the sedimented cells and frozen at $-20^{\circ} \mathrm{C}$ or below for short-term storage (up to 2 weeks) and $-70{ }^{\circ} \mathrm{C}$ or below for long-term storage (18). The H21-A5 guideline also recommends that individual laboratories should perform in-house validation studies (18).

Previous studies have focused on coagulation factor assays in fresh-frozen plasma (FFP) for transfusion (19-23). We want to accurately detect factor activities that reflect the true factor activities in the body. Furthermore, biobanks are a very important biomedical research resource and the development of biomarker detection, molecular diagnosis, translational medicine, and multidisciplinary disease research urgently needs the support of a large number of high-quality clinical biospecimens $(24,25)$. Therefore, we also wanted to provide relevant factor data for the construction and sustainable development of high-quality clinical biobanks. The important pre-analytical variables primarily comprise sample collection, transportation, storage time, and temperature. Unsuitable samples can lead to unreliable factor activities and interfere with clinical decisions. Therefore, we planned to perform in-house validation studies on pre-analytical variables for factor activity assays in our laboratory. Thus, the aims of this study were to investigate whether storage temperature $\left(4\right.$ and $25{ }^{\circ} \mathrm{C}$ ) and time influence factor activity up to $24 \mathrm{~h}$, whether freeze-thaw times influence factor activity after longterm storage, and whether changes arising from delayed analysis result in clinically relevant differences, with a view to establishing acceptable pre-analytical storage time, temperature, and freeze-thaw guidelines for our laboratory. In the present study, we determined the FII coagulation activity (FII:C), FV:C, FVII:C, FX:C, FXI:C, and FXII:C in citrate-anticoagulated plasma stored for 0 (baseline), 2 , $4,6,8,12$, and $24 \mathrm{~h}$ at 4 and $25^{\circ} \mathrm{C}$, and compared the factor activity differences for various freeze-thaw times (snapfreezing at $-80^{\circ} \mathrm{C}$ and thawing in a $37^{\circ} \mathrm{C}$ water bath).

\section{Methods}

The sample population consisted of 52 asymptomatic individuals (26 men and 26 women; mean age: 45 years; age range, 20-62 years) recruited from The First Affiliated Hospital of Zhejiang University for physical examination in October 2016. A blood sample was collected from each subject in the morning after a $12-\mathrm{h}$ fast. For this, a $5.4-\mathrm{mL}$ venous whole blood sample was collected into two tubes (2.7 mL each) containing $0.109 \mathrm{M}$ sodium citrate as an anticoagulant (Becton Dickinson, Franklin Lakes, NJ, USA) at a blood-to-anticoagulant ratio of 9:1. The 52 samples were divided into two groups and used to detect the factor stability at $-80{ }^{\circ} \mathrm{C}[30$ samples $(15$ men and 15 women) for freeze-thaw tests] or 25 and $4{ }^{\circ} \mathrm{C}$ [22 samples $(11$ men and 11 women)]. All samples were centrifuged (10 min, 3,000 ×g) to obtain fresh platelet-poor plasma. Next, 30 samples were quickly divided into four Eppendorf (EP) tubes (numbered 1-4) and capped, and the remaining 22 samples were quickly divided into $13 \mathrm{EP}$ tubes [baseline $(0 \mathrm{~h})$, plus storage for 2, 4, $6,8,12$, and $24 \mathrm{~h}$ at 25 or $4^{\circ} \mathrm{C}$, respectively] in 15 minutes. The EP tubes used for the aliquots were composed of a non-activating plastic.

The 22 samples divided into 13 EP tubes (group 1, showed in Figure 1) were tested for FII:C, FV:C, FVII:C, FX:C, FXI:C, and FXII:C immediately (baseline, $0 \mathrm{~h}$ ) and after storage for $2,4,6,8,12$, and $24 \mathrm{~h}$ at 25 or $4{ }^{\circ} \mathrm{C}$, respectively, using a Sysmex CS5100 system (Sysmex, Kobe, Japan) and Siemens reagents (Siemens, Marburg, Germany).

In the 30 samples divided into four EP tubes (group 2, showed in Figure 2), FII:C, FV:C, FVII:C, FX:C, FXI:C, and FXII:C in each No. 1 aliquot (freeze-thawed 0 times) were tested immediately (baseline, $0 \mathrm{~h}$ ), and all testing was completed within $30 \mathrm{~min}$ after sample collection. The No. 2-4 aliquots were snap-frozen at $-80{ }^{\circ} \mathrm{C}$. After $24 \mathrm{~h}$, all aliquots were thawed in a $37^{\circ} \mathrm{C}$ water bath for $6 \mathrm{~min}$ and mixed by 10 end-over-end inversions. The No. 2 aliquots (freeze-thawed one time) were tested using the same lots of reagents and same instrument as the fresh sample testing 


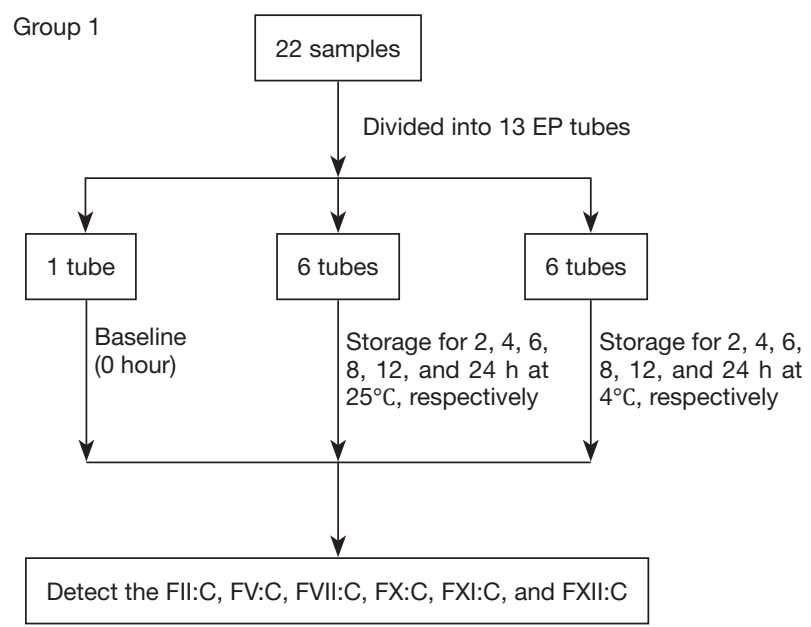

Figure 1 The flow chart of detection factors stabilities after storage for $0,2,4,6,8,12$, and $24 \mathrm{~h}$ at 25 or $4^{\circ} \mathrm{C}$.

above, while the No. 3 and 4 aliquots were re-frozen at $-80{ }^{\circ} \mathrm{C}$. After another $24 \mathrm{~h}$, the No. 3 and 4 aliquots were again thawed in a $37^{\circ} \mathrm{C}$ water bath for 6 min and mixed. The No. 3 aliquots (freeze-thawed two times) were tested as described for the No. 2 aliquots, and the No. 4 aliquots were re-frozen at $-80^{\circ} \mathrm{C}$. After another $24 \mathrm{~h}$, the No. 4 aliquots (freeze-thawed three times) were thawed in a $37{ }^{\circ} \mathrm{C}$ water bath for $6 \mathrm{~min}$, mixed, and tested as described for the No. 2 aliquots.

\section{Ethics statement}

This study was approved by the Ethics Committees of The First Affiliated Hospital of Zhejiang University (Ethical Application Ref: 2015-15). Subjects provided written informed consent for their samples to be used in the study according to the principles expressed in the Declaration of Helsinki.

\section{Laboratory assays}

The aliquots were tested for FII:C, FV:C, FVII:C, FX:C, FXI:C, and FXII:C by two-stage coagulation assays using the Sysmex CS5100 system and the following Siemens reagents: coagulation factor II-deficient plasma (lot: 503634A); coagulation factor V-deficient plasma (lot: 504951); coagulation factor VII-deficient plasma (lot: 500753A); coagulation factor X-deficient plasma (lot: 504011B); coagulation factor XI-deficient plasma (lot: 50324B); and coagulation factor XII-deficient plasma (lot:

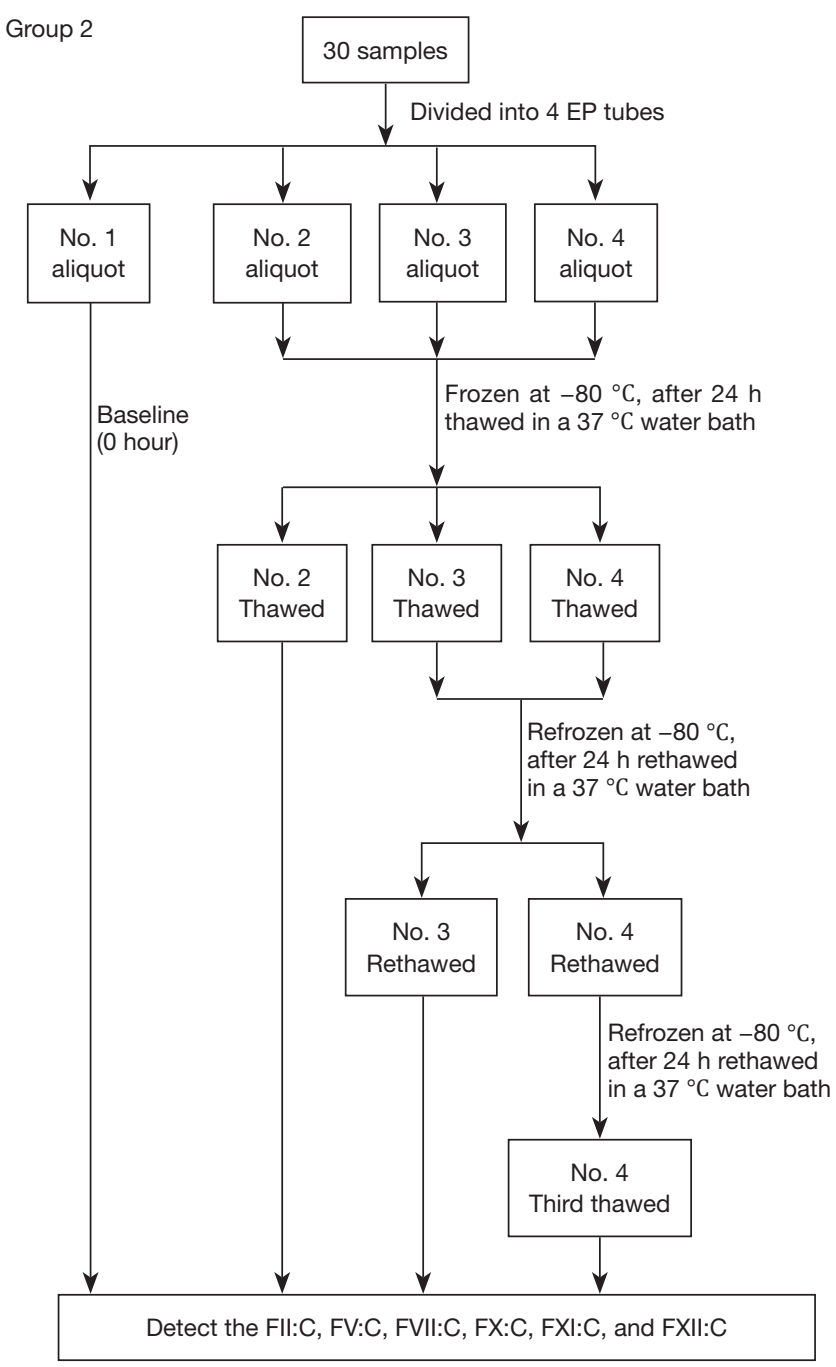

Figure 2 The flow chart of detection factors stabilities after three freeze-thawing cycles at $-80^{\circ} \mathrm{C}$.

503407A). The results were expressed as \%.

\section{Statistical analyses}

The coagulation factor activity results were reported as mean \pm standard deviation. The statistical significance of differences in values for repeated-measure multiple groups or frozen samples compared with baseline values was evaluated by repeated-measures analysis of variance (ANOVA). To assess the stability of coagulation factor activity, the percent differences were calculated by the following equation (26,27): (value at storage time $X$ - value at baseline) $\times 100 \% /$ value at baseline. In accordance with our previous study (26) and that by van Geest-Daalderop 
Table 1 Values for FII:C, FV:C, FVII:C, FX:C, FXI:C, and FXII:C in samples stored at 4 and $25^{\circ} \mathrm{C}$

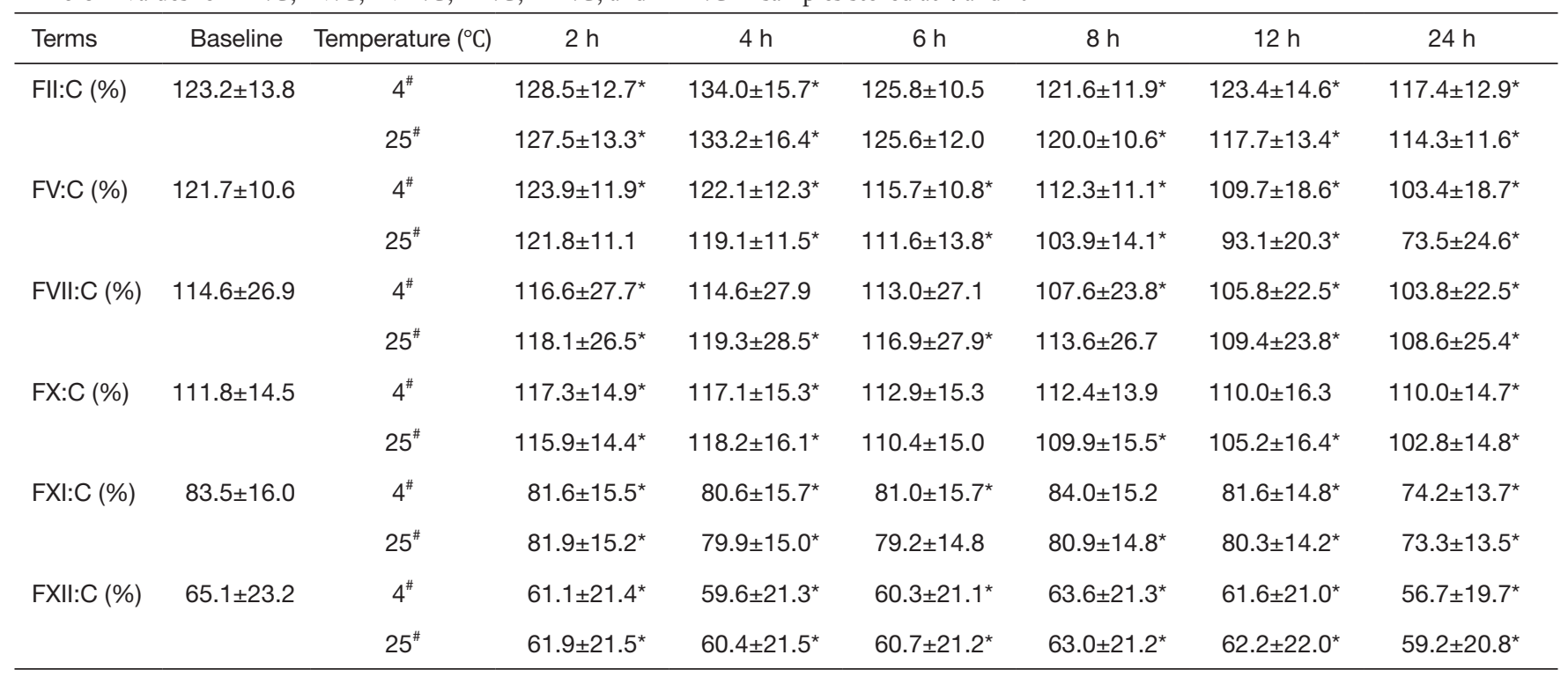

The statistical significance of differences in values for repeated-measure multiple groups compared with baseline values was evaluated by repeated-measures analysis of variance (ANOVA); ${ }^{*}, \mathrm{P}<0.05$ vs. baseline values; \#, P<0.05 among groups. FII:C, FV:C, FVII:C, FX:C, FXI:C, and FXII:C: coagulation activities of FII, FV, FVII, FX, FXI, and FXII, respectively.

et al. (27), a clinically relevant difference was defined as a mean change of $>10 \%$. If the number of individuals with $>10 \%$ changes was less than $25 \%$ of the total 22 samples, the effect was termed moderate. If more than $25 \%$ of the total 22 samples had $>10 \%$ changes, the effect was deemed large. Trend plots for the mean percent changes were created, with storage times at 25 and $4{ }^{\circ} \mathrm{C}$ on the $\mathrm{X}$-axis, and mean percent differences on the $\mathrm{Y}$-axis. Differences between the values for FII:C, FV:C, FVII:C, FX:C, FXI:C, and FXII:C before and after freeze-thawing were calculated by the following equation: (value after freeze-thawing $\mathrm{X}$ times - value at baseline) $\times 100 \% /$ value at baseline. Difference plots of the percent changes were drawn with the percent changes of all samples on the Y-axis and the freeze-thaw times on the $\mathrm{X}$-axis. These plots were visually inspected to determine the underlying variability characteristics of the relationships between factor activity at baseline and after freeze-thawing, as well as individual differences. Two dotted lines were drawn in these plots to indicate plus or minus $10 \%$ changes. The trends for mean percent differences in FII:C, FV:C, FVII:C, FX:C, FXI:C, and FXII:C in samples after freeze-thawing three times were evaluated. Values of $\mathrm{P}<0.05$ were considered statistically significant. All statistical analyses were performed using SPSS version 22 (SPSS Inc., Chicago, IL, USA).

\section{Results}

\section{Stability studies}

The results for FII:C, FV:C, FVII:C, FX:C, FXI:C, and FXII:C after storage for $2,4,6,8,12$, and $24 \mathrm{~h}$ at 4 or $25^{\circ} \mathrm{C}$ are shown in Table 1 and compared with baseline values $(0 \mathrm{~h})$. Table 2 shows the mean percent changes and acceptable stability times for FII:C, FV:C, FVII:C, FX:C, FXI:C, and FXII:C when the samples were stored under the abovementioned conditions. Figure 3 shows the percent changes and change trends for FII:C, FV:C, FVII:C, FX:C, FXI:C, and FXII:C in samples stored for 2, 4, 6, 8, 12, and $24 \mathrm{~h}$ at 4 or $25^{\circ} \mathrm{C}$, and the two dotted lines represent plus or minus $10 \%$ changes. We found that FII:C, FV:C, FVII:C, FX:C, FXI:C, and FXII:C all gradually reduced with storage time at both 4 and $25{ }^{\circ} \mathrm{C}$. Among the factors, FV:C reduced the fastest and the change reached $-60 \%$ after storage for $24 \mathrm{~h}$ at $25^{\circ} \mathrm{C}$. By combining the results, we found that the mean percent changes in FII:C, FV:C, FVII:C, FX:C, FXI:C, and FXII:C were less than $10 \%$ compared with the baseline values after storage for $8 \mathrm{~h}$ at $4{ }^{\circ} \mathrm{C}$ and $4 \mathrm{~h}$ at $25^{\circ} \mathrm{C}$. When the storage time was prolonged, the acceptable times for stability of FII:C, FV:C, FVII:C, FX:C, FXI:C, and FXII:C were $24,8,8,24,12$, and $12 \mathrm{~h}$ at $4{ }^{\circ} \mathrm{C}$ and $24,4,8,8,12$, and $12 \mathrm{~h}$ at $25^{\circ} \mathrm{C}$. Among the factors, FV:C was the most 
Table 2 Mean percent differences for FII:C, FV:C, FVII:C, FX:C, FXI:C, and FXII:C in samples stored at 4 and $25^{\circ} \mathrm{C}$

\begin{tabular}{|c|c|c|c|c|c|c|c|c|c|c|c|c|c|c|}
\hline Terms & \multicolumn{2}{|c|}{$2 \mathrm{~h}$} & \multicolumn{2}{|c|}{$4 \mathrm{~h}$} & \multicolumn{2}{|c|}{$6 \mathrm{~h}$} & \multicolumn{2}{|c|}{$8 \mathrm{~h}$} & \multicolumn{2}{|c|}{$12 \mathrm{~h}$} & \multicolumn{2}{|c|}{$24 \mathrm{~h}$} & \multicolumn{2}{|c|}{ Accept (h) } \\
\hline FII:C (\%) & 4.66 & 3.70 & 9.02 & 8.18 & 2.67 & 2.49 & -1.01 & -2.19 & 0.27 & -4.13 & -4.54 & -7.02 & 24 & 24 \\
\hline FV:C (\%) & 1.75 & 0.07 & 0.27 & -2.11 & -4.87 & $-8.16^{\star}$ & -7.72 & $-14.62^{*}$ & $-10.00^{\star}$ & $-23.62^{*}$ & $-15.30^{\star}$ & $-39.67^{*}$ & 8 & 4 \\
\hline FVII:C (\%) & 1.62 & 3.17 & -0.09 & 4.05 & -1.22 & 2.03 & -5.96 & -0.92 & $-7.22^{\star}$ & $-4.12^{\star}$ & $-8.94^{\star}$ & $-5.16^{\star}$ & 8 & 8 \\
\hline FXI:C (\%) & -2.21 & -1.73 & -3.43 & -4.21 & -2.93 & -5.00 & 0.86 & -2.90 & -1.99 & -3.51 & $-10.97^{*}$ & $-12.04^{*}$ & 12 & 12 \\
\hline FXII:C (\%) & -5.91 & -4.70 & -8.43 & -7.22 & -7.06 & -6.53 & -4.22 & -1.65 & -2.58 & -4.08 & $-12.40^{*}$ & $-8.29^{\star}$ & 12 & 12 \\
\hline
\end{tabular}

Changes were calculated according to the following formula: (value at storage time $X-$ value at baseline) $\times 100 \% / v a l u e$ at baseline; ${ }^{*}$, changes of $>10 \%$ in individual samples occurred in $>25 \%$ of samples. FII:C, FV:C, FVII:C, FX:C, FXI:C, and FXII:C: coagulation activities of FII, FV, FVII, FX, FXI, and FXII, respectively.
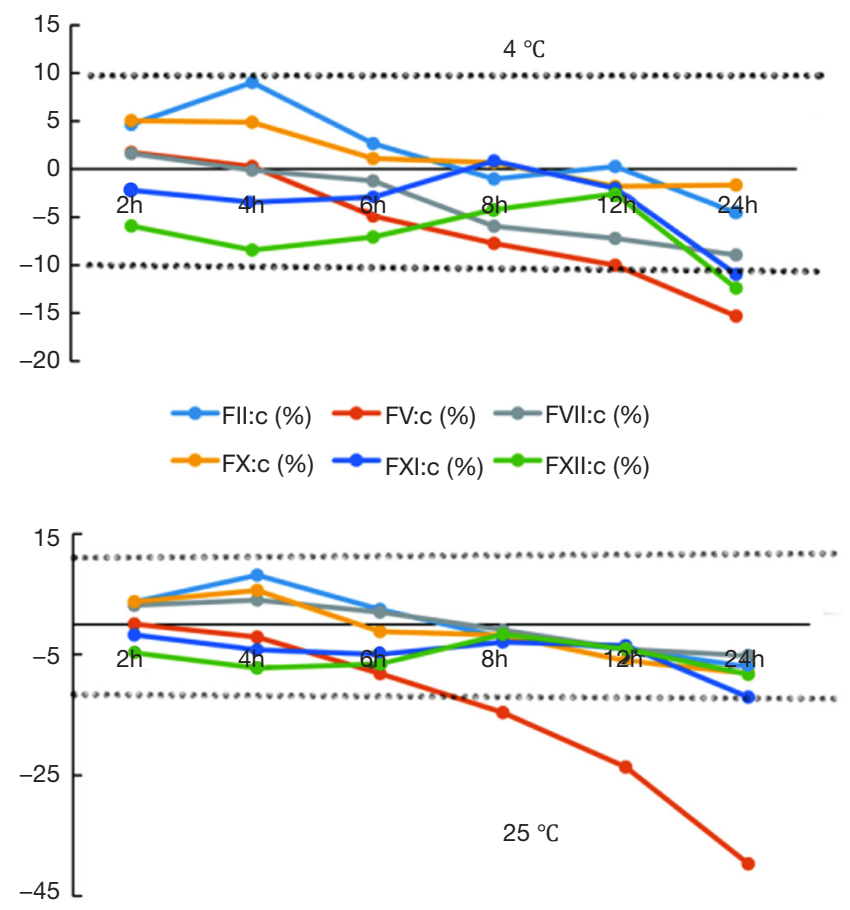

Figure 3 Trend plots for mean percent changes in FII:C, FV:C, FVII:C, FX:C, FXI:C, and FXII:C in samples stored for 2, 4, 6, 8,12 , and 24 h at 4 and $25^{\circ} \mathrm{C}$. The two dotted lines show plus or minus $10 \%$ changes.

unstable, and the stability of FV:C and FX:C at $4{ }^{\circ} \mathrm{C}$ was better than that at $25^{\circ} \mathrm{C}$.

\section{Freeze-thaw results}

The baseline results and all freeze-thaw results for FII:C,
FV:C, FVII:C, FX:C, FXI:C, and FXII:C are shown in Table 3, and the differences between the baseline and freeze-thaw values are shown in Table 4. Figure 4 shows the individual differences in FII:C, FV:C, FVII:C, FX:C, FXI:C, and FXII:C after freeze-thawing with storage at $-80{ }^{\circ} \mathrm{C}$, and the two dotted lines represent plus or minus $10 \%$ changes. In this figure, individual samples with changes of $>10 \%$ can be visualized from the scatter plots, and the numbers of samples with changes of $>10 \%$ in each group can be visualized to determine moderate or large effects. The results showed that the acceptable freeze-thaw times for FII:C, FV:C, FVII:C, FX:C, FXI:C, and FXII:C were $2,2,3,3,2$, and 1 , respectively.

\section{Discussion}

Coagulation factor assays are very important for diagnosing, treating, and monitoring inherited and acquired bleeding disorders $(1,14)$. Therefore, it is necessary to evaluate the effects of pre-analytical variables for sample storage. Our study is the first to investigate the effects of split-tube storage for $2,4,6,8,12$, and $24 \mathrm{~h}$ at 25 and $4^{\circ} \mathrm{C}$, and to evaluate the effects of freeze-thaw storage at $-80{ }^{\circ} \mathrm{C}$ on FII:C, FV:C, FVII:C, FX:C, FXI:C, and FXII:C in fresh citrate-anticoagulated plasma.

In our study, although some significant differences were observed between baseline factor activities and activities with different storage times, temperatures, and freezethaw times, the differences were within the acceptable time frames. A clinically relevant difference was defined as a mean change of $>10 \%$, and changes of $>10 \%$ in individual samples occurred in $>25 \%$ of samples. We found that FII:C, 
Table 3 Values for FII:C, FV:C, FVII:C, FX:C, FXI:C, and FXII:C in samples subjected to freeze-thawing at $-80{ }^{\circ} \mathrm{C}$

\begin{tabular}{|c|c|c|c|c|c|}
\hline Terms & 0 & 1 & 2 & 3 & $P$ \\
\hline FV:C (\%) & $124.8 \pm 19.5$ & $117.3 \pm 18.3^{\star}$ & $117.6 \pm 17.6^{*}$ & $111.7 \pm 18.4^{*}$ & $<0.001$ \\
\hline FVII:C (\%) & $107.3 \pm 20.7$ & $102.8 \pm 21.0^{*}$ & $101.9 \pm 19.0^{*}$ & $99.8 \pm 18.2^{*}$ & $<0.001$ \\
\hline FX:C (\%) & $104.6 \pm 17.8$ & $102.5 \pm 16.3^{\star}$ & $103.2 \pm 17.9^{*}$ & $100.0 \pm 17.3^{*}$ & $<0.001$ \\
\hline FXII:C (\%) & $68.5 \pm 19.3$ & $68.2 \pm 19.1$ & $61.5 \pm 17.7^{*}$ & $62.2 \pm 18.2^{*}$ & $<0.001$ \\
\hline
\end{tabular}

The statistical significance of differences in values for frozen samples compared with baseline values was evaluated by repeatedmeasures analysis of variance (ANOVA); ${ }^{*}, \mathrm{P}<0.05$ vs. baseline results. 0 , freeze-thawed 0 times; 1 , freeze-thawed one time; 2 , freezethawed two times; 3, freeze-thawed three times. FII:C, FV:C, FVII:C, FX:C, FXI:C, and FXII:C: coagulation activities of FII, FV, FVII, FX, FXI, and FXII, respectively.

Table 4 Acceptable freeze-thaw times for FII:C, FV:C, FVII:C, FX:C, FXI:C, and FXII:C in samples subjected to freeze-thawing at $-80{ }^{\circ} \mathrm{C}$

\begin{tabular}{|c|c|c|c|c|}
\hline Terms & d1 & d2 & d3 & Accept \\
\hline $\mathrm{FV}: \mathrm{C}(\%)$ & $-5.28(-12.54$ to -1.45$)$ & $-5.43(-15.22$ to 0$)$ & $-9.69(-23.82 \text { to }-3.69)^{\star}$ & Twice \\
\hline FVII:C (\%) & $-4.65(-12.78$ to 5.36$)$ & $-4.9(-8.69$ to 0.62$)$ & $-7.24(-11.82$ to -2.19$)$ & Thrice \\
\hline FX:C (\%) & $-1.67(10.79$ to 5.96$)$ & $-1.38(-5.5$ to 4.83$)$ & $-4.08(-13$ to 4.17$)$ & Thrice \\
\hline FXII:C (\%) & $0(-11.12$ to 5.51$)$ & $-8.6(-20.64 \text { to }-1.38)^{*}$ & $-10.19(-20.99 \text { to }-1.65)^{*}$ & Once \\
\hline
\end{tabular}

*, more than $25 \%$ of samples had $>10 \%$ changes. d1, difference $1=$ (value for freeze-thawing one time - value at baseline) $\times 100 \% / v a l u e$ at baseline; d2, difference 2 = (value for freeze-thawing two times - value at baseline) $\times 100 \% /$ value at baseline; d3, difference $3=($ value for freeze-thawing three times - value at baseline) $\times 100 \%$ value at baseline; accept, acceptable freeze-thaw times; FII:C, FV:C, FVII:C, FX:C, FXI:C, and FXII:C: coagulation activities of FII, FV, FVII, FX, FXI, and FXII, respectively.

FV:C, FVII:C, FX:C, FXI:C, and FXII:C all gradually reduced at both 4 and $25^{\circ} \mathrm{C}$. When the storage time was prolonged, the acceptable short-term storage times for stability of FII:C, FV:C, FVII:C, FX:C, FXI:C, and FXII:C were $24,8,8,24,12$, and $12 \mathrm{~h}$ at $4{ }^{\circ} \mathrm{C}$ and $24,4,8,8,12$, and $12 \mathrm{~h}$ at $25^{\circ} \mathrm{C}$. We also determined the acceptable freeze-thaw times for FII:C, FV:C, FVII:C, FX:C, FXI:C, and FXII:C. Through our in-house validation study, we established suitable storage temperatures and times for factor activity assays in the short term. If the factor activity cannot be detected within these acceptable time frames, we suggest that plasma should be frozen at $-80{ }^{\circ} \mathrm{C}$ for transportation and/or storage, and thawed for activity detection at appropriate times, because the changes in FVII:C and FX:C in plasma freeze-thawed three times, FII:C, FV:C, and FXI:C in plasma freeze-thawed two times, and FXII:C in plasma freeze-thawed one time compared with baseline values were all less than $10 \%$.

No previous studies have analyzed the stability of coagulation factor activities in fresh citrate-anticoagulated plasma besides our early research, in which we determined the effects of storage time and temperature on FVIII:C and FIX:C in fresh plasma, and found that samples for FVIII:C and FIX:C can be safely stored for $\leq 2$ and $\leq 4 \mathrm{~h}$ at both 4 and $25^{\circ} \mathrm{C}(26)$. Heil et al. (28) found the stability in plasma (allowing for $10 \%$ variation from the initial value) was 48 hours for factor $\mathrm{V}$ in healthy subjects at $6{ }^{\circ} \mathrm{C}$ and for 8 hours at room temperature. Woodhams et al. (29) reported the frozen stability of factors II, V, VII, VIII, IX, X, XI, and XII and found all factors (allowing for 10\% variation) were stable for up to 3 months if frozen at $-24{ }^{\circ} \mathrm{C}$ or lower, and for at least 18 months at $-74{ }^{\circ} \mathrm{C}$.

True activity determination of factors in fresh plasma from patients is very valuable in clinical applications. In 


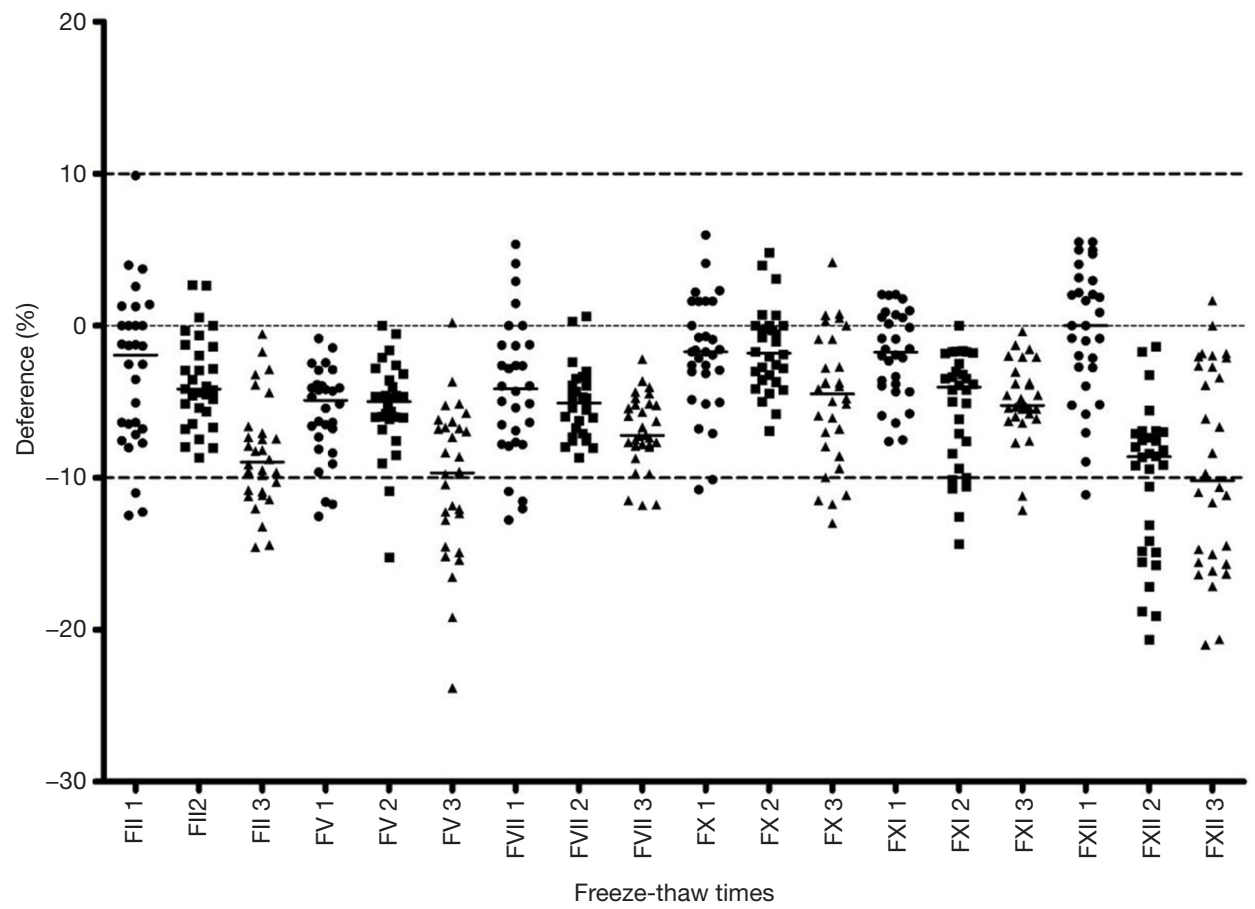

Figure 4 Freeze-thaw difference plots for FII:C, FV:C, FVII:C, FX:C, FXI:C, and FXII:C. •: 1, percent changes in FII:C, FV:C, FVII:C, FX:C, FXI:C, and FXII:C after freeze-thawing one time; $\mathbf{~ : ~ 2 , ~ p e r c e n t ~ c h a n g e s ~ i n ~ F I I : C , ~ F V : C , ~ F V I I : C , ~ F X : C , ~ F X I : C , ~ a n d ~ F X I I : C ~ a f t e r ~}$ freeze-thawing two times; $\boldsymbol{\Delta}:$ 3, percent changes in FII:C, FV:C, FVII:C, FX:C, FXI:C, and FXII:C after freeze-thawing three times. The two dotted lines show plus or minus $10 \%$ changes.

the present study, we found that all factor activities were significantly reduced with extended storage time up to $24 \mathrm{~h}$ at 25 and $4{ }^{\circ} \mathrm{C}$. To avoid the factor activities becoming lower than the true activities, we established suitable storage times and temperatures for each factor in our laboratory: the storage times for FII:C, FV:C, FVII:C, FX:C, FXI:C, and FXII:C were $24,8,8,24,12$, and $12 \mathrm{~h}$ at $4^{\circ} \mathrm{C}$ and $24,4,8,8$, 12 , and $12 \mathrm{~h}$ at $25^{\circ} \mathrm{C}$, respectively. When the factor activity cannot be determined within these acceptable time frames, we recommend that plasma should be frozen and thawed at appropriate times for analysis. Our study provides reliable data for clinical diagnosis, treatment, and monitoring of bleeding disorders.

Our study has some limitations. First, our study was based on asymptomatic individuals. Thus, we did not study the factor activities in patients with oral anticoagulant therapy, hepatitis, liver cirrhosis, liver failure, and haemophilia, and cannot comment on the factor stability in these situations. Second, we used only the CS5100 system and Siemens reagents to detect the factor activities with coagulation assays. Third, our study was a single-centre study and based solely on the Chinese Han population in Zhejiang province. Our findings need to be validated by further research based on multi-centre studies, with different study populations, disease states, reagents, instruments, and determination methodologies.

\section{Acknowledgements}

We thank Richard Robins, PhD, from Liwen Bianji, Edanz Editing China (www.liwenbianji.cn/ac), for editing the English text of a draft of this manuscript.

Funding: This work was financially supported by grants from the Science Foundation of Health Bureau of Zhejiang Province (2015KYA086 and 2014KYA252) and the Zhejiang Provincial Natural Science Foundation of China (LY15H190002).

\section{Footnote}

Conflicts of Interest: The authors have no conflicts of interest to declare. 
Ethical Statement: This study was approved by the Ethics Committees of The First Affiliated Hospital of Zhejiang University (Ethical Application Ref: 2015-15). Subjects provided written informed consent for their samples to be used in the study according to the principles expressed in the Declaration of Helsinki.

\section{References}

1. Levin M, Potter GK, Shah MS. Review and consideration of coagulopathies. Clin Podiatr Med Surg 1998; 15:499-512.

2. Salaj P. Congenital and acquired bleeding disorders. Vnitr Lek 2018;64:547-58.

3. Coppola A, Tagliaferri A, Santoro C, et al. Alloantibodies and Congenital Bleeding Disorders: New Insights in the Pathogenesis and Management. Semin Thromb Hemost 2018;44:505-8.

4. Liu W, Xuan M, Xue F, et al. Acquired coagulation factor $\mathrm{X}$ deficiency: three cases report and literature review. Zhonghua Xue Ye Xue Za Zhi 2014;35:633-6.

5. Asselta R, Peyvandi F. Factor V deficiency. Semin Thromb Hemost 2009;35:382-9.

6. Mariani G, Bernardi F. Factor VII Deficiency. Semin Thromb Hemost 2009;35:400-6.

7. Menegatti M, Peyvandi F. Factor X deficiency. Semin Thromb Hemost 2009;35:407-15.

8. Clarkson K, Rosenfeld B, Fair J, et al. Factor XI deficiency acquired by liver transplantation. Ann Intern Med 1991;115:877-9.

9. Osborn NK, Ustundag Y, Zent CS, et al. Factor XII deficiency acquired by orthotopic liver transplantation: case report and review of the literature. Am J Transplant 2006;6:1743-5.

10. Lippi G, Favaloro EJ, Montagnana $M$, et al. Inherited and acquired factor $\mathrm{V}$ deficiency. Blood Coagul Fibrinolysis 2011;22:160-6.

11. Pasmant E, Dumont B, Lacapere JJ, et al. A severe neonatal presentation of factor II deficiency. Eur J Haematol 2011;87:464-6.

12. Kerr R. New insights into haemostasis in liver failure. Blood Coagul Fibrinolysis 2003;14 Suppl 1:S43-5.

13. Saner FH, Kirchner C. Monitoring and Treatment of Coagulation Disorders in End-Stage Liver Disease. Visc Med 2016;32:241-8.

14. Meeks SL, Abshire TC. Abnormalities of prothrombin: a review of the pathophysiology, diagnosis, and treatment. Haemophilia 2008;14:1159-63.
15. Guo H, Na X, Hou L, et al. Classifying Chinese Questions Related to Health Care Posted by Consumers Via the Internet. J Med Internet Res 2017;19:e220.

16. LV J. Improvement of Hierarchical Diagnosis and Treatment System Under Deepening Medical and Health Reform. Chinese Hospital Management 2014;34:1-3.

17. Song C, Shan ZM, Zhang WM. A nationwide investigation and analysis of present status of independent clinical laboratories in China and suggestions. Chin J Hosp Admin 2011;27:288-91.

18. CLSI. Collection, Transport, and Processing of Blood Specimens for Testing Plasma-Based Coagulation Assays and Molecular Hemostasis Assays; Approved Guideline. 5th ed. CLSI Document H21-A5. Wayne, PA, 2008.

19. Ben-Tal O, Zwang E, Eichel R, et al. Vitamin K-dependent coagulation factors and fibrinogen levels in FFP remain stable upon repeated freezing and thawing. Transfusion 2003;43:873-7.

20. Contreras M, Ala FA, Greaves M, et al. Guidelines for the use of fresh frozen plasma. British Committee for Standards in Haematology, Working Party of the Blood Transfusion Task Force. Transfus Med 1992;2:57-63.

21. Koerner K, Stampe D. Stability of blood coagulation factors in deep frozen fresh plasma by storage at -20 degrees $\mathrm{C}$ and -40 degrees C. Infusionsther Klin Ernahr 1984;11:46-50.

22. Kellner S, Stocker U, Furst G. Stability of blood coagulation factors in plasma frozen 6 and 18 hours after blood collection. Infusionsther Klin Ernahr 1985;12:208-10.

23. Buchta C, Felfernig M, Höcker P, et al. Stability of coagulation factors in thawed, solvent/detergent-treated plasma during storage at 4 degrees $C$ for 6 days. Vox sanguinis 2004;87:182-6.

24. Zander J, Bruegel M, Kleinhempel A, et al. Effect of biobanking conditions on short-term stability of biomarkers in human serum and plasma. Clin Chem Lab Med 2014;52:629-39.

25. Zika E, Paci D, Braun A, et al. A European survey on biobanks: trends and issues. Public Health Genomics 2011;14:96-103.

26. Feng L, Zhao Y, Zhao H, et al. Effects of storage time and temperature on coagulation tests and factors in fresh plasma. Sci Rep 2014;4:3868.

27. van Geest-Daalderop JH, Mulder AB, Boonman-de Winter LJ, et al. Preanalytical variables and off-site blood collection: influences on the results of the prothrombin time/international normalized ratio test and implications 
for monitoring of oral anticoagulant therapy. Clin Chem 2005;51:561-8.

28. Heil W, Grunewald R, Amend M, et al. Influence of time and temperature on coagulation analytes in stored plasma.

Cite this article as: Zhao Y, Feng G, Feng L. Effects of preanalytical storage time, temperature, and freeze-thaw times on coagulation factors activities in citrate-anticoagulated plasma. Ann Transl Med 2018;6(23):456. doi: 10.21037/atm.2018.11.24
Clin Chem Lab Med 1998;36:459-62.

29. Woodhams B, Girardot O, Blanco MJ, et al. Stability of coagulation proteins in frozen plasma. Blood Coagul Fibrinolysis 2001;12:229-36. 